\title{
Antimicrobial prescribing in the surgical and medical wards at a private hospital in KwaZulu-Natal, South Africa, 2019
}

\author{
V T Jacob, ${ }^{1}$ BPharm; S Mahomed, ${ }^{2}$ MB ChB, Dip HIV Man (SA), FCPHM, MMed (Public Health Medicine), PhD \\ ${ }^{1}$ School of Health Sciences, University of KwaZulu-Natal, Durban, South Africa \\ ${ }^{2}$ School of Laboratory Medicine and Medical Sciences, University of KwaZulu-Natal, Durban, South Africa
}

Corresponding author: VT Jacob (valencia.jacob@lifehealthcare.co.za)

\begin{abstract}
Background. Appropriate antimicrobial use is imperative owing to the misuse of antimicrobials, which has resulted in a growing burden of antimicrobial resistance. Evidence-based guidelines should be adhered to in order to ensure the sustainability of effective antimicrobials. Objectives. To assess the appropriateness of antimicrobial prescribing at a private hospital in Durban, KwaZulu-Natal, South Africa.

Methods. The records of patients admitted to the surgical and medical wards over a 2-month period were reviewed to assess the choice, dose and duration of prescribed antimicrobials. The guidelines used to determine the appropriateness of the prescriptions were: the Standard Treatment Guidelines and Essential Medicines List for South Africa - Hospital Level Adults, 2015 edition, the antimicrobial indications and prescribing instructions in the South African Medicines Formulary, the South African Antibiotic Stewardship Programme guidelines and the evidence-based surgical prophylaxis guidelines adopted by the hospital group where the research was conducted.

Results. During the study period, 466 patients were admitted and prescribed an antimicrobial, of whom $220(47.2 \%)$ were admitted to the surgical ward and $246(52.8 \%)$ to the medical wards. A total of 779 antimicrobials were prescribed. Of the 660 antimicrobials prescribed for empirical treatment, 305 (46.2\%) were appropriately prescribed based on drug choice, dose and duration. Of the 38 antimicrobials that were classified as targeted, 36 (94.7\%) were prescribed at the correct dose and 33 (86.8\%) for the correct duration. Of the 81 antimicrobials prescribed for surgical prophylaxis, only $32(39.5 \%)$ met the criteria for appropriateness in terms of drug choice, dose and duration.

Conclusions. Our findings suggest that compliance with evidence-based guidelines for the use of antimicrobials is not optimal in hospitals in the private sector. Antimicrobials are prescribed inappropriately for empirical treatment and for surgical prophylaxis. Private hospital groups should consider adopting antimicrobial prescribing guidelines that are mandatory for doctors to adhere to in order to promote rational antimicrobial prescribing, and thereby reduce the burden of antimicrobial resistance.
\end{abstract}

S Afr Med J 2021;111(6):582-586. https://doi.org/10.7196/SAMJ.2021.v111i6.15403

The threat of antimicrobial resistance is paramount in the area of infectious diseases due to its rapid spread on a global scale. The occurrence of multidrug-resistant micro-organisms in hospitals is linked to an increased risk of patient morbidity and mortality, and longer hospital stays. ${ }^{[1]}$ The continuing emergence of antimicrobial-resistant bacteria resulting from the misuse and abuse of antimicrobials has become a major public health problem, with $>1$ of 3 antimicrobial prescriptions in the emergency department being inappropriate. ${ }^{[2,3]}$ Without co-ordinated and immediate action on a global scale, the world is moving towards a post-antibiotic era in which common infections could once again kill. ${ }^{[4]}$

The profuse use of broad-spectrum antimicrobials is influenced by the lack of timely detection of causative micro-organisms and their antimicrobial susceptibility patterns. This use has caused rapid increases in emerging bacterial resistance. ${ }^{[5]}$ Antimicrobial de-escalation, which refers to narrowing of the antimicrobial spectrum based on sensitivity of the pathogen, is a commonly accepted management strategy in critically ill patients. ${ }^{[6]}$ The antimicrobial spectrum should be narrowed as soon as possible, based on the clinical condition of the patient, the pathogen identified and the sensitivity profile obtained from the antibiogram. When no evidence of bacterial infection is present, antimicrobial therapy should be suspended. ${ }^{[7]}$ However, de-escalation has not been widely adopted, which could be due to the doctor's hesitancy to change an antimicrobial that has demonstrated effectiveness, an inadequate understanding of how to de-escalate appropriately, lack of microbiological data, and different opinions about the safety and usefulness of de-escalation. ${ }^{[6]}$

Antimicrobial surgical prophylaxis forms part of a collection of processes that aim to decrease the incidence of surgical site infections (SSIs). One of the fundamental goals of surgical prophylaxis is to reduce the bacterial load in the wound, thus assisting the natural defences of the host to prevent the occurrence of infection. ${ }^{[8]}$ The World Health Organization (WHO) has developed a surgical safety checklist intended to improve the safety of patients undergoing surgical procedures, as safety measures are often not appropriately applied. ${ }^{\left[{ }^{[9]}\right.}$ The suitable use of surgical prophylaxis can significantly decrease the rate of SSIs by up to $50 \% \cdot{ }^{[9]}$ Evidence-based guidelines can be followed to prevent SSIs, while also avoiding the emergence of antimicrobial resistance. Research has shown that better compliance with guidelines for antimicrobial surgical prophylaxis is required. ${ }^{[10]}$

In 2015, South Africa (SA) responded to the WHO's request to raise awareness regarding the preservation of antimicrobials through appropriate and rational use by implementing World Antibiotic Awareness Week initiatives. ${ }^{[1]}$ The development of the Standard Treatment Guidelines (STG) and Essential Medicines List (EML) for South Africa - Hospital Level Adults, 2015 edition, ${ }^{[12]}$ forms part of a plan to minimise the unnecessary prescription of antimicrobials in the public sector. In the private sector, formularies are developed according to hospital management or hospital group protocols; however, antimicrobial prescribing is rarely standardised in practice. The use of formularies can be used to regulate the choice of 
antimicrobials, thereby reducing expenses to the patient, the hospital and the government. ${ }^{[13]}$ Antimicrobial prescription practices can only be improved when there is evidence that there are deficiencies in practices. Therefore, the objectives of this study were to investigate the appropriateness of antimicrobial prescribing in terms of dose, duration and frequency; to determine whether antimicrobial prescribing is adjusted based on microscopy, culture and sensitivity results; and to assess the appropriateness of antimicrobials used for surgical prophylaxis.

\section{Methods}

\section{Study setting}

The study was conducted at a private hospital in Durban, KwaZuluNatal, SA. The hospital provides 215 beds and serves a middleincome population.

\section{Study population and sample frame}

Patients admitted to the surgical and medical wards over a 2-month period in 2019, and who were prescribed an antimicrobial during their admission, were considered for inclusion in this study. Each ward is a 27-bed unit. The surgical ward includes the following: general surgery, plastic surgery, urology and orthopaedic surgery patients, and some patients in other disciplines.

Patients who were prescribed antimicrobials for tuberculosis or for the eradication of Helicobacter pylori were excluded. Antivirals were excluded, with the exception of oseltamivir, as the patient sample was taken during the influenza season. Patients $<18$ years of age, patients with incomplete or missing data and those who were admitted for gynaecological conditions were also excluded. Only the first admission was included for patients who were readmitted during the study period.

\section{Sample size}

Approximately 150 - 170 patients are admitted to the surgical and medical wards per month. Based on previous admissions, 40 - 65\% of patients are prescribed an antimicrobial per month. A sample size of 385 was estimated to be effective for this study. This sample size produces a two-sided $95 \%$ confidence interval (CI), with a precision of $\sim 7.5 \%$, where variability is unknown. The calculation is based on normal distribution and the assumption that there would be $>30$ patients.

\section{Study design}

This was an observational, analytical cross-sectional study.

\section{Data collection and analysis}

Electronic patient records were used to collect clinical and patient data, such as age and gender. The clinical variables included diagnosis, whether a patient had a comorbidity, length of hospital stay, ward type (surgical or medical) and discipline. The variables that were related to antimicrobial prescription included antimicrobial choice, route of administration, dose and duration, and microscopy, culture and sensitivity test results. The infection markers assessed were procalcitonin (PCT), C-reactive protein (CRP), erythrocyte sedimentation rate (ESR) and white cell count (WCC). All patient data were anonymised and no patient identifiers were collected.

A distinction was made between patients who were prescribed antimicrobials for prophylaxis and those who were prescribed antimicrobials as treatment for an infection. In terms of therapeutic use, antimicrobials were further classified as empirical or targeted. An antimicrobial was classified as targeted if it was prescribed according to antibiogram results. Antimicrobials were classified as prophylactic if the patient records reflected that the antimicrobial was used for prophylaxis or if it was administered 1 hour prior to the start of a surgical procedure.

Antimicrobial prescriptions were assessed according to drug choice, dose and duration to ascertain if these complied with the STG and $E M L,{ }^{[12]}$ the South African Medicines Formulary ${ }^{[14]}$ and the South African Antibiotic Stewardship Programme guidelines (a pocket guide to antibiotic prescribing for adults in South Africa, 2014). ${ }^{[15]}$ The antimicrobials that were prescribed for surgical prophylaxis were assessed according to the surgical prophylaxis guidelines adopted by the hospital group where the research was conducted. Antimicrobial therapy was classified as appropriate if the drug choice, dose and duration were consistent with the guidelines. The assessment of appropriateness was performed by the senior pharmacist. Any inconsistencies between the guidelines were discussed with the hospital clinical practice pharmacist to reach consensus. There were no queries that required further input from a clinician.

Quantitative data were summarised using the mean and standard deviation (SD). Categorical variables were presented using proportions. The odds ratio (OR) was calculated to assess associations between length of hospital stay and the presence of microscopy, culture and sensitivity testing. The $\chi^{2}$ test was used to assess for significance of associations between categorical variables, and the Kruskal-Wallis test to determine differences between $\geq 3$ groups. A $p$-value of $<0.05$ was considered statistically significant.

\section{Ethical considerations}

Gate-keeper permission was obtained from the hospital manager and pharmacy manager at the facility. Permission was also obtained from the Health Research Ethics Committee of the hospital group. Ethical approval for the study was granted by the Biomedical Research Ethics Committee, University of KwaZulu-Natal (ref. no. BE457/19).

\section{Results}

A total of 1051 patients were admitted over the 2-month period, of whom 601 (57.2\%) were prescribed antimicrobials. After applying the exclusion criteria, 466 patients were included in the study, of whom 246 (52.8\%) were from the medical wards and 220 (47.2\%) from the surgical ward.

\section{Profile of patients}

Just over half of the study population $(n=253 ; 54.3 \%)$ were female patients (Table 1). Of the 246 patients admitted to the medical wards, there were more female $(n=154 ; 62.6 \%)$ than male patients. The converse was seen in the surgical ward, where only 99 of 220 patients $(45 \%)$ were female $(p<0.001)$. Overall, the mean age of female patients was 51.8 (SD 16.6) years and the mean age of males was $52.8(17.3)$ years $(p=0.56)$. In the medical wards, the mean age for females was 53.2 (15.4) years compared with 50.0 (17.6) years for males $(p=0.14)$. In the surgical ward, the mean age for females was 49.8 (16.8) years compared with 54.8 (16.7) years for males $(p=0.03)$.

Infection markers were requested for $278(59.7 \%)$ patients, of whom 127 (45.7\%) were from the medical wards. More than half of these patients $(n=162 ; 58.3 \%)$ had elevated infection markers. A comorbidity was recorded in 276 patients (59.2\%). The median length of stay was 4 (range 1 - 41) days. More than a third of patients $(n=160 ; 34.3 \%)$ had a length of stay $>4$ days.

\section{Antimicrobial prescribing}

A total of 779 antimicrobials were prescribed over the 2-month period, of which $660(84.7 \%)$ were prescribed empirically, 81 (10.4\%) 


\begin{tabular}{ll} 
Table 1. Profile of patients in the surgical and medical wards \\
at a private hospital in KwaZulu-Natal, 2019 \\
\hline \begin{tabular}{l} 
Patient profile \\
\hline Age, years
\end{tabular} \\
$18-20$ & Frequency (\%) \\
$21-30$ & $12(2.6)$ \\
$31-40$ & $49(10.5)$ \\
$41-50$ & $59(12.7)$ \\
$51-60$ & $87(18.6)$ \\
$61-70$ & $108(23.2)$ \\
$>70$ & $80(17.2)$ \\
Gender & $71(15.2)$ \\
Female & \\
Male & $253(54.3)$ \\
Length of stay, days & $213(45.7)$ \\
$1-2$ & $122(26.2)$ \\
$3-4$ & $184(39.5)$ \\
$>4$ & $160(34.3)$
\end{tabular}

were prescribed for surgical prophylaxis and 38 (4.9\%) were targeted. More than half of the antimicrobials $(n=422 ; 54.2 \%)$ were prescribed in the medical wards - the antimicrobial most frequently prescribed in these wards was amoxicillin/clavulanic acid $(n=80 ; 19 \%)$. In the surgical ward, the antimicrobial most frequently prescribed was cefuroxime $(n=75 ; 21 \%)$. Just less than a third of the antimicrobials were prescribed by a physician $(n=142 ; 30.8 \%)$ and $92(19.7 \%)$ by a general practitioner (Table 2). Among all patients, the most common antimicrobials empirically prescribed were amoxicillin/clavulanic acid $(n=126 ; 19 \%)$, ceftriaxone $(n=106 ; 16 \%)$ and cefuroxime $(n=82$; $12.4 \%)$. The most common antimicrobials prescribed for surgical prophylaxis were amoxicillin/clavulanic acid $(n=21 ; 25.9 \%)$, cefazolin $(n=17 ; 21 \%)$ and cefuroxime $(n=13 ; 16 \%)$. Of the 71 patients who were prescribed oseltamivir, only 27 were tested for the influenza virus, of whom 11 tested positive for a strain of influenza.

\section{Appropriateness of antimicrobial prescribing}

Of a total of 779 antimicrobials prescribed, 407 (52.3\%) met the criteria for appropriate drug choice, 734 (94\%) were prescribed using the appropriate dose and $560(71.9 \%)$ were prescribed using the appropriate duration. There was no significant difference in the appropriateness of antimicrobial prescribing between the surgical and medical wards $(p=0.5)$ (Fig. 1). Of the 81 antimicrobials prescribed for surgical prophylaxis, $42(51.9 \%)$ met the criteria for appropriate drug choice, 77 (95\%) were prescribed at the correct dose and $45(55.6 \%)$ were prescribed for the correct duration. Overall, 32 (39.5\%) of these antimicrobials met the criteria for appropriateness in terms of drug choice, dose and duration.

\section{De-escalation}

Microscopy, culture and sensitivity tests were ordered for 159 (34.1\%) patients; a pathogen was cultured from 77 (48.4\%). Of these 77 patients, only $28(36.4 \%)$ were de-escalated appropriately. Patients with a length of stay $>4$ days were significantly more likely to have a microscopy, culture and sensitivity test done than those with a length of stay of 1 - 2 days (OR $2.4 ; p<0.05$ ) or 3 - 4 days (OR 1.8 ; $p<0.05$ ).

\section{Discussion}

This is the first reported study of antimicrobial prescribing in the surgical and medical wards at a private hospital in KwaZulu-Natal. More than half of the patients in this study were prescribed an

\begin{tabular}{|c|c|}
\hline Antimicrobial prescribing & Frequency (\%) \\
\hline \multicolumn{2}{|l|}{ Antimicrobial } \\
\hline Amikacin & $25(3.2)$ \\
\hline Amoxicillin & $2(0.3)$ \\
\hline Amoxicillin/clavulanic acid & $153(19.6)$ \\
\hline Azithromycin & $30(3.9)$ \\
\hline Cefazolin & $17(2.2)$ \\
\hline Cefpodoxime & $7(0.9)$ \\
\hline Ceftazidime & $2(0.3)$ \\
\hline Ceftriaxone & $116(14.9)$ \\
\hline Cefuroxime & $99(12.7)$ \\
\hline Ciprofloxacin & $35(4.5)$ \\
\hline Clarithromycin & $16(2)$ \\
\hline Clindamycin & $23(3)$ \\
\hline Doxycycline & $2(0.3)$ \\
\hline Ertapenem & $16(2)$ \\
\hline Fosfomycin & $2(0.3)$ \\
\hline Levofloxacin & $81(10.4)$ \\
\hline Linezolid & $2(0.3)$ \\
\hline Meropenem & $6(0.8)$ \\
\hline Metronidazole & $33(4.2)$ \\
\hline Moxifloxacin & $28(3.6)$ \\
\hline Nitrofurantoin & $2(0.3)$ \\
\hline Norfloxacin & $1(0.1)$ \\
\hline Oseltamivir & $71(9.1)$ \\
\hline Piperacillin/tazobactam & $5(0.6)$ \\
\hline Teicoplanin & $2(0.3)$ \\
\hline Trimethoprim/sulfamethoxazole & $1(0.1)$ \\
\hline Vancomycin & $2(0.3)$ \\
\hline \multicolumn{2}{|l|}{ Route of administration } \\
\hline Oral & $275(35.3)$ \\
\hline Intravenous & $504(64.7)$ \\
\hline \multicolumn{2}{|l|}{ Discipline } \\
\hline Medical & $142(30.8)$ \\
\hline General practitioner & $92(19.7)$ \\
\hline Urology & $76(16)$ \\
\hline General surgery & $57(12.2)$ \\
\hline Gastrointestinal & $38(8.1)$ \\
\hline Other & $19(4)$ \\
\hline Plastic surgery & $17(3.6)$ \\
\hline Orthopaedic & $12(2.6)$ \\
\hline Ear/nose/throat & $10(2.1)$ \\
\hline Dental/maxillofacial & $3(0.6)$ \\
\hline
\end{tabular}

antimicrobial during their hospital stay. While there are no published reports with which to compare our findings, the antimicrobial prescribing in this study is lower than the $61 \%$ reported in an intensive care unit (ICU) setting in a hospital in the private sector in SA. ${ }^{[16]}$ In our study, $94 \%$ of antimicrobials were prescribed using the appropriate dose. This is similar to findings from the aforementioned study, where $91 \%$ of antimicrobials were prescribed using the appropriate dose. ${ }^{[16]}$

An alarming $52.6 \%$ of antimicrobials were inappropriately prescribed according to drug choice, dose and duration. A study conducted in 8 primary care facilities in the Cape Town Metro District also showed that non-adherence to national guidelines was $54.9 \%{ }^{[17]}$ The guideline used was the STG and EML - Primary Health Care Level, 2014 edition. The most common reason for non- 


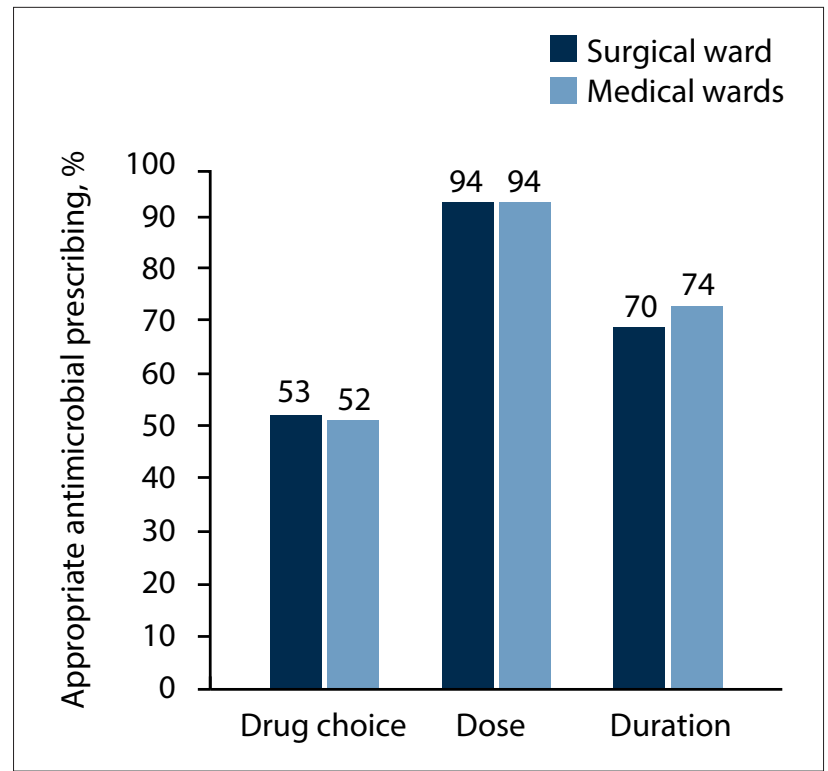

Fig. 1. Appropriateness of antimicrobial prescribing in the surgical and medical wards at a private hospital in KwaZulu-Natal, Durban, South Africa, 2019.

adherence in this study was because a diagnosis was not specified in the medical records. In our study, all the relevant information was collected electronically and the diagnosis was always noted, as it is a requirement that the ICD-10 coding system is used for all patients. In an Australian study that evaluated the appropriateness of antimicrobial prescribing in private hospitals, it was found that $48 \%$ of antimicrobials were inappropriate. ${ }^{[18]}$ The proportion of inappropriately prescribed antimicrobials in our study is also similar to previous studies conducted in Turkey (47\%) and Namibia (38\%). ${ }^{[19,20]}$ In the current study, 52.3\% of antimicrobials met the criteria for appropriate drug choice. This is lower than the results from the WHO pilot study in Brits, North West Province, where $67 \%$ of 2370 prescriptions complied with STG and EML guidelines for appropriate drug choice. ${ }^{[21]}$ This pilot study was conducted in the public sector; therefore, the difference in appropriateness of drug choice could be due to the private sector having an array of antimicrobials to choose from, with poor adherence to formularies.

Microscopy, culture and sensitivity tests were done for $34 \%$ of patients in our study, and only $36 \%$ of those who had a pathogen cultured were de-escalated appropriately. In an SA study, commonly known as the Prevalence of Infection in SA (PISA) intensive care unit study, de-escalation was practised in $33.3 \%$ and $19.7 \%$ of public and private sector patients, respectively. ${ }^{[22]}$ The difference in proportion between the public and private sector can be attributed to the presence of 'open' ICUs in the private sector, where each patient is managed by the admitting doctor, who may prescribe antimicrobials indiscriminately, often without appropriate knowledge of the epidemiology of the unit. ${ }^{[22]}$ In a study conducted in a private hospital in SA in 2015, de-escalation was noted in only $13.1 \%$ of patients in the ICU. ${ }^{[16]}$ In our study, the higher proportion of patients in whom the antimicrobial was de-escalated compared with patients in other private hospitals in SA, may be due to the incorporation of a regional clinical pharmacist to guide the antimicrobial stewardship programme at our hospital. Since the aforementioned study was conducted in 2015, it is possible that de-escalation in the private sector has improved as antimicrobial stewardship efforts have developed over the last few years. Further studies in the private sector are required to determine if this is indeed the case. In a study that measured de-escalation in a private acute care hospital in North Carolina, USA, antimicrobial regimens were de-escalated in $63 \%$ of patients. ${ }^{[23]}$ This high proportion may be attributed to the establishment of antimicrobial stewardship programmes at the hospital more than a decade before the study was conducted. In contrast, the antimicrobial stewardship programme in our study setting was established 5 years ago and is still facing challenges. Antimicrobial stewardship meetings are meant to be held quarterly, with representation from at least one doctor. It is also preferable for a microbiologist to attend these meetings; however, the attendance of doctors and microbiologists is a rare occurrence.

We found that $<40 \%$ of the antimicrobials prescribed for surgical prophylaxis met the criteria for appropriateness in terms of drug choice, dose and duration. In a systematic review of 18 studies that evaluated the adherence to guidelines for antimicrobial surgical prophylaxis, substantial differences were observed in all the outcomes that were assessed. ${ }^{[10]}$ These outcomes included administration of an antimicrobial at the correct time (12.7 - 100\%), correct antimicrobial choice $(22$ - 95\%) and adequate antimicrobial prophylaxis (0.3 $84.5 \%){ }^{[10]}$ The findings from the systematic review and our study indicate that greater adherence to guidelines for surgical prophylaxis is necessary. The dose was correct in $95 \%$ of the antimicrobials prescribed for surgical prophylaxis, indicative that the drug choice and the duration contributed to the overall high level of inappropriate prescribing. In our study setting, surgical prophylaxis is monitored and audited regularly and doctors should be contacted when patients are maintained on antimicrobial prophylaxis for a prolonged period. The inappropriate duration of antimicrobials prescribed for surgical prophylaxis is due to doctors not discontinuing the antimicrobial timeously. The hospital hosts an ageing population of doctors and it is plausible that there is reluctance to change prescribing habits. Factors such as age, gender, educational status, specialty and work experience have been noted to influence doctors' prescription patterns. ${ }^{[24]}$ In a study that investigated prescription patterns in Chinese county hospitals, it was found that doctors $<45$ years of age prescribed fewer antimicrobials than those $>45$ years old. ${ }^{[2]}$ In a study in Ethiopia, $76 \%$ of antimicrobials prescribed for surgical prophylaxis were administered for $>24$ hours, only $10.6 \%$ of the drug choices complied with the American Society of Health-System Pharmacists guideline and none of the selections complied with the national standard treatment guidelines of the country. ${ }^{[25]}$ The failure to comply with the guidelines is the result of prolonged surgical prophylaxis administration beyond the recommended timeframe. Although the guidelines state that administration of the antimicrobial used for surgical prophylaxis should be stopped within 24 hours, $44.4 \%$ of the antimicrobials were administered for up to 5 days ${ }^{[25]} \mathrm{A}$ study in Johannesburg that assessed anaesthetists' knowledge of surgical prophylaxis found that overall knowledge was poor, with few anaesthetists reporting to follow any guideline. ${ }^{[26]}$ The mean knowledge score was $56.2 \%$, and only $36.3 \%$ of participants were aware that most guidelines state that prophylaxis should be continued only for the duration of the surgery. ${ }^{[26]}$

In a study to describe SA prescribers' knowledge of, attitudes to and perceptions of antimicrobial resistance, it was found that prescribers of antimicrobials in the private sector in SA felt pressure from patients to prescribe antimicrobials, even though they are conscious of the problem of antimicrobial resistance. ${ }^{[27]}$ This factor may contribute to the high proportion (52.6\%) of antimicrobials that were inappropriately prescribed in our study. In a review of 33 studies, the factors that influenced the prescribing decisions of physicians were evaluated, and many factors related to the working and external environment were identified. These included physicians' personal attributes, cost of the medicine and marketing and promotion strategies of the pharmaceutical industry. ${ }^{[28]}$ These factors showed that 
prescribing is not always purely for the benefit of the patient, but can also be guided by individual interest. The use of suitable and reliable practice guidelines could decrease the harmful impact of certain factors and endorse rational prescribing. ${ }^{[28]}$

Our study was conducted in only one private hospital, which limited the generalisability of the findings. However, in the private sector in SA, doctors often admit and manage patients at more than one hospital; therefore, our findings are likely to reflect the practices at other hospitals in the private sector, particularly in Durban. Data were collected retrospectively using an electronic surveillance program; therefore, clinical data such as the patient's temperature and radiological findings could not be assessed. It was only noted whether a patient had a comorbidity - the type of comorbidity was not recorded. It is possible that the presence of comorbidities may have affected the choice, dose and duration of the antimicrobial. Furthermore, the assessment of infection markers was based only on whether the patient had a raised PCT, CRP, ESR or WCC, with no alignment of the patient's clinical condition. The appropriateness of antimicrobial selection was based on the assumption that the electronically recorded diagnosis was accurate. A prospective study with the collection of more clinical data and inclusion of clinicians may provide better insight regarding reasons for antimicrobial choices, doses and duration.

\section{Conclusions}

Our findings suggest that compliance with evidence-based guidelines for the use of antimicrobials is not optimal in hospitals in the private sector. Antimicrobials are prescribed inappropriately for empirical treatment and for surgical prophylaxis. There is an urgent need to cascade antimicrobial prescribing guidelines using innovative educational strategies to target all prescribing doctors working in the private sector. Private hospital groups should consider adopting antimicrobial prescribing guidelines that are mandatory for doctors to adhere to. Doctors should be informed of the antimicrobial and surgical prophylaxis prescribing guidelines when applying to work at a private hospital, and sign an agreement to adhere to these guidelines. Restrictions on the empirical use of certain antimicrobials, and regular audits of antimicrobial prescribing with feedback to prescribers, should also be implemented to promote rational antimicrobial prescribing.

\section{Declaration. None.}

Acknowledgements. None.

Author contributions. VTJ conceptualised the research, collected and analysed the data, and wrote all versions of the manuscript. SM supervised all aspects of the research and reviewed all versions of the manuscript.

Funding. VTJ is currently employed by the hospital group, and has obtained funding towards completion of a postgraduate degree. The hospital group did not contribute to the study design, data collection, methods, analysis and interpretation of the results, and writing of the manuscript.

Conflicts of interest. None.
1. La Fauci V, Alessi V. Antibiotic resistance: Where are we going? Ann Ig 2018;30(4):52-57. https:// doi.org/10.7416/ai.2018.2235

2. Komolafe O. Antibiotic resistance in bacteria - an emerging public health problem. Malawi Med J 2004;15(2):6367. https://doi.org/10.4314/mmj.v15i2.10780
.

3. Denny KJ, Gartside JG, Alcorn K, Cross JW, Maloney S, Keijzers G. Appropriateness of antibiotic prescribing in the emergency department. J Antimicrob Chemother 2019;74(2):515-520. https:// doi.org/10.1093/jac/dky447

4. World Health Organization. Global action plan on AMR. 2018. https://www.who.int/antimicrobialresistance/global-action-plan/en/ (accessed 27 November 2018).

5. Akova M. Epidemiology of antimicrobial resistance in bloodstream infections. Virulence 2016;7(3):252-266. https://doi.org/10.1080\%2F21505594.2016.1159366

6. Garnacho-Montero J, Escoresca-Ortega A, Fernández-Delgado E. Antibiotic de-escalation in the ICU. Curr Opin Infect Dis 2015;28(2):193-198. https://doi.org/10.1097/qco.0000000000000141

7. Silva BN, Andriolo RB, Atallah AN, Salomão R. De-escalation of antimicrobial treatment for adults with sepsis, severe sepsis or septic shock. Cochrane Database Syst Rev 2013;(3):CD007934. https:// doi.org/10.1002\%2F14651858.CD007934.pub3

8. Gouvêa $\mathrm{M}$, Novaes $\mathrm{C}$ de $\mathrm{O}$, Iglesias AC. Assessment of antibiotic prophylaxis in surgical patients at the Gaffrée e Guinle University Hospital. Rev Col Bras Cir 2016;43(4):225-234. https://doi. at the Gaffree e Guinle University
org/10.1590/0100-69912016004001

9. World Health Organization. Guidelines for safe surgery 2009: Safe surgery saves lives. 2009. http:// . World Health Organization. Guidelines for safe surgery 2009: Safe surgery saves lives. 2009.
whqlibdoc.who.int/publications/2009/9789241598552_eng.pdf (accessed 14 August 2020).

10. Gouvêa M, Novaes C, Pereira D, Iglesias A. Adherence to guidelines for surgical antibiotic prophylaxis: A review. Braz J Infect Dis 2015;19(5):517-524. https://doi.org/10.1016/j. bjid.2015.06.004

11. South African Government. Health on antibiotic resistance. 2019. https://www.gov.za/speeches health-describes-antibioticsantimicrobial-resistance-looming-public-health-catastrophe-18 (accessed 5 May 2019).

2. National Department of Health. Standard Treatment Guidelines and Essential Medicines List: Hospital Level Adults. 4th ed. Pretoria: NDoH, 2015.

13. Essack S, Schellack N, Pople T, et al. GARP: Antibiotic supply chain and management in human health. S Afr Med J 2011:101(8):562-566.

14. Rossiter D, Blockman M, Barnes KI, eds. South African Medicines Formulary. 13th ed. Cape Town: Health and Medical Publishing Group, 2019.

15. Wasserman S, Boyles T, Mendelson M. A pocket guide to antibiotic prescribing for adults in South Wasserman S, Boyles T, Mendelson M. A pocket guide to antibiotic prescribing for adults in South
Africa. 2014. South African Antibiotic Stewardship Programme (SAASP). 2020. https://www.fidssa. Africa. 2014. South African Antibiotic Stewardship Programme (SAASP). 2020. https://wWw.fid
co.za/Content/Documents/SAASP_Antibiotic_Guidelines_2015.pdf (accessed 11 March 2019).

16. Chunnilall D, Peer A, Naidoo I, Essack S. An evaluation of antibiotic prescribing patterns in adult intensive care units in a private hospital in KwaZulu-Natal. South Afr J Infect Dis 2015;30(1):17-22. https://doi.org/10.1080/23120053.2015.1103956

17. Gasson J, Blockman M, Willems B. Antibiotic prescribing practice and adherence to guidelines in primary care in the Cape Town Metro District, South Africa. S Afr Med J 2018:108(4):304. https:// doi.org/10.7196/samj.2017.v108i4.12564

18. Cotta MO, Robertson MS, Upjohn LM, Marshall C, Liew D, Buising KL. Using periodic pointprevalence surveys to assess appropriateness of antimicrobial prescribing in Australian private hospitals. Intern Med J 2014;44(3):240-246. https://doi.org/10.1111/imj.12353

19. Ceyhan M, Yildirim I, Ecevit C, et al. Inappropriate antimicrobial use in Turkish pediatric hospitals: A multicenter point prevalence survey. Int J Infect Dis 2010;14(1):e55-e61. https://doi.org/10.1016/j. A multicenter po 2009.03 .013

20. Nakwatumbah S, Kibuule D, Godman B, et al. Compliance to guidelines for the prescribing of antibiotics in acute infections at Namibias national referral hospital: A pilot study and the implications. Expert Rev Anti-Infect Ther 2017;15(7):713-721. https://doi.org/10.1080/14787210.2017.1320220

Expert Rev Anti-Infect Ther 2017;15(7):713-721. https://doi.org/10.1080/14787210.2017.1320220
21. World Health Organization. Community-based surveillance of antimicrobial use and resistance in 21. World Health Organization. Community-based surveillance of antimicrobial use and resistance in
resource-constrained settings. 2009. https://www.who.int/medicines/publications/who_emp_2009.2/ resource-constrained settings. 2009.
en/ (accessed 23 September 2020).

22. Paruk F, Richards G, Scribante J, Bhagwanjee S, Mer M, Perrie H. Antibiotic prescription practices and their relationship to outcome in South Africa: Findings of the Prevalence of Infection in South African intensive care units (PISA) study. S Afr Med J 2012;102(7):613. https://doi.org/10.7196/ samj. 5833

23. Liu P, Ohl C, Johnson J, Williamson J, Beardsley J, Luther V. Frequency of empiric antibiotic deescalation in an acute care hospital with an established antimicrobial stewardship program. BMC Infect Dis 2016;16(1):751. https://doi.org/10.1186/s12879-016-2080-3

24. Wang H, Li N, Zhu H, Xu S, Lu H, Feng Z. Prescription pattern and its influencing factors in Chinese county hospitals: A retrospective cross-sectional study. PLoS ONE 2013;8(5):e63225. https://doi. county hospitals: A retrospective
org/10.1371/journal.pone. 0063225

25. Alemkere G. Antibiotic usage in surgical prophylaxis: A prospective observational study in the surgical ward of Nekemte referral hospital. PLoS ONE 2018;13(9):e0203523. https://doi. org/10.1371/journal.pone. 0203523

26. Jocum J, Lowman W, Perrie H, Scribante J. Anaesthetists' knowledge of surgical antibiotic prophylaxis: A prospective descriptive study. South Afr J Anaesth Analg 2018;24(4):97-102. https:// doi.org/10.1080/22201181.2018.1487634

27. Farley E, Stewart A, Davies M, Govind M, van den Bergh D, Boyles T. Antibiotic use and resistance: Knowledge, attitudes and perceptions among primary care prescribers in South Africa. S Afr Med J 2018;108(9):763. https://doi.org/10.7196/samj.2018.v10819.12933

28. Davari M, Khorasani E, Tigabu BM. Factors influencing prescribing decisions of physicians: A review. Ethiop J Health Sci 2018;28(6):795-804. https://doi.org/10.4314\%2Fejhs.v28i6.15

Accepted 13 January 2021. 